ORIGINAL ARTICLE

\title{
Accidents don't happen any more: junior doctors' experience of fatal accident inquiries in Scotland
}

\author{
J McCulloch, M Sykes, F Haut
}

Postgrad Med J 2005;81:185-187. doi: 10.1136/pgmj.2004.019430

See end of article for authors' affiliations

.....................

Correspondence to: Dr J McCulloch, Elmwood, Royal Cornhill Hospital, 26 Cornhill Road, Aberdeen AB25 2ZH, Scotland: justine.mcculloch@nhs.net

Submitted 30 January 2004 Accepted 13 April 2004
Objective: To determine the experience of junior doctors cited as witnesses at fatal accident inquiries (FAls).

Design: Retrospective questionnaire study.

Setting and subjects: 40 junior doctors who had been involved in FAls from January 1998 to August 2002 were identified by the Medical Protection Society and Medical and Dental Defence Union of Scotland: 21 completed questionnaires were returned, a response rate of $52.5 \%$.

Results: The process and objectives of FAls were poorly understood. Few participants were proactive in contacting their defence union. There was a perceived lack of both formal and informal supports with respondents describing a "blame culture" within the medical profession. The experience of the FAl was generally considered stressful with respondents describing possible improvements relating to support, information, training, and the FAl procedure.

Conclusions: Junior medical staff are poorly informed in this medicolegal area. There is a need for the development of mechanisms both to support junior doctors and to ensure that adverse incidents are dealt with using modern risk management techniques to minimise the risk of recurrence.
$\mathrm{T}$ he process of public inquiry into "sudden, suspicious and unexplained deaths and deaths occurring in circumstances giving rise to serious public concern"1 differs among Scotland, Northern Ireland, and England and Wales. The Scottish legal system uses fatal accident inquiries (FAIs), which are analogous to coroners inquiries in England and Wales, but held on a statutory basis. FAIs in regard of medical care are held at the discretion of the Lord Advocate, in the "public interest". The role of the FAI is neither to determine guilt nor primarily to apportion blame; rather it is to determine where, when, and how the death took place and what could have been done to prevent it. ${ }^{\prime}$

This legal remit exists in a socio-political environment increasingly intolerant of "accidents" as events of innocent origin. $^{2}$ Indeed, the validity of the very term "accident" has been questioned. ${ }^{3}$ It is within this environment that research has identified FAIs as negative experiences for the medical profession. ${ }^{4}$ It has been suggested that the stress of such adverse incidents may be considerable, resulting in psychological consequences including suicidal thoughts or actions. ${ }^{5}$ The phenomenon of "closing ranks" has also been described. ${ }^{6}$ It is against this background that the importance of support from colleagues and partners has been emphasised. ${ }^{67}$

For those doctors involved in an FAI, there is little in the literature to inform as to the mechanics of the process, the professional supports available, or the similar experiences of their peers. Aitkenhead has identified deficiencies in training and management action plans resulting in adverse consequences for relatives and hospital staff.

This study aims to identify empirically the experience of junior doctors who have been through a FAI; to learn from their experience and inform future practice.

\section{METHODS}

The Crown Office ${ }^{7}$ was approached to identify all junior doctors involved in FAIs throughout Scotland over the specified period but was unable to assist, stating its data systems were not suited to the purpose. The medical defence organisations were therefore contacted and the two largest in
Scotland, the Medical Protection Society (MPS) and the Medical and Dental Defence Union of Scotland (MDDUS) agreed to distribute an anonymous questionnaire to junior doctors cited as witnesses in FAIs from January 1998 to August 2002.

The questionnaire (available from authors) was based on a review of the literature, consultation with psychiatric colleagues and the MPS, and a feasibility study. It comprised four sections examining the following areas: demographics and clinical experience; details relating to the process from the adverse event itself to the FAI; the FAI itself, and the impact this had on the individual doctor. It was not possible to generate a suitable comparison group. None the less, findings from this group are important irrespective of whether they differ from other doctors.

\section{RESULTS}

\section{Demographics and experience}

The MPS and MDDUS identified 40 junior doctors who had been involved in FAIs over the period January 1998 to August 2002. All junior doctors received an initial mailing and then a follow up request six months later; it was not possible to contact non-responders because of the anonymous distribution method. Twenty one completed questionnaires were returned over a 12 month period, representing a response rate of $53 \%$ ( $29 \%$ were members of the MDDUS; $71 \%$ MPS). The sex ratio was 33\% (male):67\% (female). Most respondents had trained at Scottish universities and 10 (49\%) had been registered medical practitioners for five years or less at the time of the FAI; a further four (19\%) were 6-10 years after registration. The median age at the time of the FAI was 31 years (range 24-46 years). Respondents currently worked within a variety of specialties with mental health (six, 29\%) and accident and emergency (three, 14\%) most represented. Experience in the various specialties at the time of the FAI

Abbreviations: FAl, fatal accident inquiry; MPS, Medical Protection Society; MDDUS, Medical and Dental Defence Union of Scotland 
ranged from 1 month to 10 years; eight (38\%) had one year or less years experience in the speciality.

\section{Process leading to the FAI}

Most respondents $(13,62 \%)$ did not have the process and probable sequence of events explained. Most participants (16, $76 \%$ ) said they received no support from management and eight $(38 \%)$ said their main support had been informal (family), five (24\%) described a complete absence of support. Colleagues were seen as unsupportive in seven (33\%) cases. Only one respondent was suspended from work. There was no formal debriefing in most cases $(15,71 \%)$.

Most staff contacted their defence union when they were informed of the FAI; four participants did so in anticipation. All participants considered their defence union to have acted in their best interests.

Seventy one per cent (15) of respondents reported that their cases had received media publicity: of these 53\% (8) found this invasive and $47 \%$ (7) thought the media portrayal of events unrepresentative.

The median time scale from the adverse incident to the FAI was 13 months (range 6-31 months).

Participants ranked the distress they experienced in the prelude to the FAI on a Likert scale $(0=$ no distress; $10=$ severe distress). The median rating was 8 (range $0-10)$; one participant did not respond.

\section{Experience at the FAl}

The duration of witness testimonies varied; the median time spent giving evidence was three hours (range five minutes to seven hours). Fifty two per cent (11) of junior doctors were required to give evidence on more than one occasion that involved a range of attendance at court; median two days (range 0.5 days to 16 days). Participants ranked the distress level of appearing in court on a Likert scale $(0=$ no distress; $10=$ severe distress) giving a median rating of six (range 2-10); three participants did not respond.

\section{Impact of FAI on professional and personal life}

Most respondents $(15,71 \%)$ felt the precipitating incident and resultant FAI affected them professionally. Respondents endorsed statements relating to: propensity to over cautiousness in decision making $(13,62 \%)$, decreased self confidence $(12,57 \%)$, and preoccupation with the adverse event and how it could have been prevented $(7,33 \%)$. Most $(13,62 \%)$ considered they had changed their practice as a result, and nearly a third $(6,29 \%)$ reported a change to their career path.

Most respondents $(15,71 \%)$ also felt the FAI had affected their personal life characterised by reduced self confidence (13, 62\%), poor sleep (11, 52\%), irritability (10, 48\%), preoccupation $(10,48 \%)$, low $\operatorname{mood}(10,48 \%)$, reduced enjoyment of activities, (10,48\%) and family problems (6, $29 \%)$.

\section{Open text comments}

Participants were asked how the process could be improved. They highlighted three areas: support, information, and improvements to the FAI itself.

\section{Support}

Respondents described a lack of support from colleagues, including consultants; from trust management and from their legal representatives. The following quotes have been truncated to protect anonymity:

"Colleagues who were present were unsupportive. I had no debriefing afterwards".
"Support from the management or Trust would have helped and also some information about the process". "There was an in-hospital inquiry, which I was not informed of and was not asked to attend and only made aware of when a colleague let the information slip".

"More support from supervisory staff and work colleagues in the medical profession."

"Support from the Hospital Trust where the incident happened"

"The Consultants didn't talk to us about it at all. They had meetings with their lawyers prior to the murder trial".

"Once the FAl was announced the situation was very distressing as there was no support from work".

"To have more contact with our legal representatives prior to $\mathrm{FAl}^{\prime \prime}$.

"Had to counsel and support junior from another specialty as she had no support and was going to quit".

\section{Information}

Respondents reported a lack of awareness of the purpose and process of the FAI and highlighted deficiencies in information sharing.

"I was not aware that what I was saying on the phone would later be used in the FAl".

"I feel I should have been informed of what was occurring prior to receiving my summons to attend".

"Being kept in the loop by management and treated as a respected colleague rather than a liability."

"More information ...awareness of counselling i.e. BMA etc."

"Not having any previous experience therefore did not know what to expect".

"Sitting in on a court case to see who people are may have helped".

\section{Potential improvements to the FAl process}

Respondents identified four main areas of difficulty: delays intrinsic to the process; the adversarial nature of giving testimony; a perceived need for debriefing after the court appearance, and issues around media publicity. The delays referred to: the timeframe between the incident and the FAI; dates for court appearances being changed at short notice, and the waiting time before being called to the witness stand.

\section{DISCUSSION}

This study found most junior doctors felt they would have benefited from discussion and education in regard to the objectives and process of the FAI at the time of the adverse incident and support over the period from the adverse incident to the inquiry and beyond. Few participants recognised the need to inform their defence union immediately, waiting until they had been contacted by the procurator fiscal. This may represent avoidance, which is a well recognised phenomenon in those who experience a traumatic event. ${ }^{8}$ The time period between the incident and the FAI was greater than one year in $57 \%$ of FAIs in this sample; potentially raising doubt as to the accuracy of a person's recollection of events. ${ }^{9}$ Of note, this duration of delay may represent an increase on previous levels. ${ }^{10}$

The study also emphasises the importance of support from colleagues, notably consultants, during what is clearly an extremely distressing period. Previous studies have highlighted the perception of a "blame culture" with reference to "witch burning" and "scapegoats" ${ }^{\prime 4}$ and these findings were 
replicated in this study. It is noteworthy that the concept of "scapegoating" or the "blame of front line individuals" is integral to the "vulnerable system syndrome": a cluster of organisational abnormalities considered to render some systems more liable to adverse events. ${ }^{11}$

Thus it is probable that the high reported distress levels felt in the period before the FAI are multifactorial in origin. Of interest, previous work in the field of trauma has shown the presence of post-traumatic stress reactions to be associated with resistance to re-examination. ${ }^{12}$ The number of subjects in this study is small because of the limited number of junior doctors who are called as witness at FAIs. However, it is possible that the respondents may represent a subgroup of doctors who are more able to tolerate the rigours of revisiting their experience despite the high distress ratings and there is a further subgroup more debilitated by the experience. ${ }^{13}$ Furthermore, the higher number of female respondents is unexplained. There were no significant differences between male and female respondents.

The authors suggest a need for the identification and development of both formal and informal support mechanisms for staff, including those involved in adverse events. Previous authors have recommended steps after death or serious injury including defence union involvement, debriefing, and possibly counselling of the whole team. ${ }^{5}$ We also suggest clinical supervision and appraisal offer additional opportunities. It is of interest that despite the current controversy surrounding debriefing ${ }^{14}{ }^{15}$ a number of respondents highlight this as something possibly helpful. More informal strategies include increasing the understanding of modern risk management, such that teams appreciate that the errors of one member could easily be those of another, moving away from the "person approach" where accidents are deemed to occur as the result of unsafe individuals, rather than unsafe systems. ${ }^{16}{ }^{17}$

\section{Limitations of the study}

The authors acknowledge limitations of this study that should be borne in mind when considering the findings. The questionnaires were distributed by the MPS and MDDUS, precluding respondents otherwise represented. The sample size is limited because of the small numbers of junior doctors involved in such inquiries. For pragmatic reasons the study could not be extended. It proved difficult to identify juniors involved in FAIs and the "good will" of the defence societies in this regard underpins the study. The FAIs concerned were held between 1998 and 2002, with the latency to FAI ranging from 6 to 31 months. It is therefore possible that the retrospective, self reported recollections and perceptions have changed in the time between the incident and the questionnaire." The anonymous nature of the questionnaire did not permit comparison between respondents and non-respondents, or comparison between the findings of the FAI and the responses, including those relating to whether the respondents' practice had changed.

\section{ACKNOWLEDGEMENTS}

We thank the MPS and the MDDUS for their invaluable assistance with this project and Dr Alistair M Hull for his help with an earlier draft of this paper.

\section{Authors' affiliations}

J McCulloch, F Haut, Carseview Centre, Ninewells Medipark, Dundee, Scotland

M Sykes, TARPC, Tayside Centre for General Practice, Dundee, Scotland

Funding: none.

Conflicts of interest: none declared.

\section{REFERENCES}

1 Fatal Accidents and Sudden Deaths Inquiry (Scotland) Act 1976

2 Holbrook J. The criminalisation of fatal medical mistakes. BMJ 2003;327: $1118-19$

3 Davies RM, Pless B. BMJ bans accidents. BMJ 2001;322:1320-1.

4 Alexander DA, Klein S, Gray NM, et al. Suicide by patients: questionnaire study of its effects on consultant psychiatrists. BMJ 2000;320:1571-4.

5 Aitkenhead A. Anaesthetic disasters: handling the aftermath. Anaesthesia 1997; 52:477-82.

6 Donaldson L. Doctors with problems in an NHS workforce. BMJ 1994:308:1277-82

7 McCulloch J, Hull AM. Fatal accident inquiries and how to survive them. BMJ Career Focus 2004;328:216-17.

8 Gelder M, Dennis G, Mayou R, et al. Reactions to stressful experiences. In: Oxford textbook of psychiatry. 3rd ed. Oxford: Oxford University Press, 1996:134-59.

9 Koriat A, Goldsmith M, Pansky A. Toward a psychology of memory accuracy. Annu Rev Psychol 2000;51:481-537.

10 Anderson S, Leitch S, Warner S. Public interest and private grief: a study of fatal accident inquiries in Scotland. Edinburgh: The Scottish Office Central Research Unit, 1995.

11 Reason J, Carthey J, de Leval MR. Diagnosing vulnerable system syndrome: an essential prerequisite to effective risk management. Quality in Health Care 2001;10:21-5.

12 Weisaeth L. Importance of high response rates in traumatic stress research. Acta Psychiatr Scand 1989;80:131-7.

13 Weisaeh L. Stress reactions in an industrial accident. [Doctoral dissertation]. Norway: Oslo University, 1984.

14 Morris PLP. Is consensus about debriefing possible? In: Raphael, Wilson. Psychological debriefing: theory, practice and evidence. Cambridge: Cambridge University Press, 2000:321-6.

15 Conlon L, Fahy TJ. Psychological debriefing for acute trauma-a welcome demise? Ir J Psychiatr Med 2001:18:43-4.

16 Reason J. Human errors: models and management. BMJ 2000;320:768-70.

17 Berwick DM. Escape fire: lessons for the future of health care. (Edited version of the plenary address delivered at the Institute for Healthcare Improvement's 11 th annual national forum). New York: The Commonwealth Fund, 2002. 